\title{
LOCAL UNCERTAINTY IN BINARY TOMOGRAPHIC RECONSTRUCTION
}

\author{
László Varga, László G. Nyúl, Antal Nagy, Péter Balázs \\ Department of Image Processing and Computer Graphics \\ University of Szeged \\ Árpád tér 2 \\ H-6720 Szeged, Hungary \\ email: [vargalg, nyul, nagya, pbalazs]@inf.u-szeged.hu
}

\begin{abstract}
We describe a new approach for the uncertainty problem arising in the field of discrete tomography, when the low number of projections does not hold enough information for an accurate, and reliable reconstruction. In this case the lack of information results in uncertain parts on the reconstructed image which are not determined by the projections and cannot be reliably reconstructed without additional information. We provide a method that can approximate this local uncertainty of reconstructions, and show how each pixel of the reconstructed image is determined by a set of given projections. We also give experimental results for validating our approach.
\end{abstract}

\section{KEY WORDS}

binary tomography, reconstruction, optimization, uncertainty

\section{Introduction}

Transmission tomography $[1,2]$ is the reconstruction of objects from their projections. This is usually done by exposing one side of the objects to some electromagnetic or particle radiation, and measuring the loss of energy at the other side. With this information one can derive the integrals of the densities of the object on the paths of the beams and gain information of the inner structure of object without making severe damage.

In discrete tomography $[3,4]$ one also assumes that the object to be reconstructed contains only few (say, 2-6) types of materials, and in the special case called binary tomography we only want to detect the presence or absence of one single material at different parts. With this prior information, algorithms were constructed capable of reconstructing objects from very few projections.

On the other hand, the reconstructions are usually affected by several types of errors, which can degrade the accuracy of the results. Such errors commonly come from a stochastic noise in the measured data caused by the nature of the projection acquisition processes, or the simplifications in the formulation of the reconstruction problem.

Also, in some applications of transmission tomography it might be necessary to reduce the number of projections, because they can damage the objects of study, or have a high cost. This lack of information can bring us to the problem, where the reconstruction is not unique, and several solutions are possible, some of which can be quite dissimilar to the desired image of the object of interest.

Finally, if we have an adequate projection set, we can still find ourselves against computational limitations, since even in the binary case, the discrete reconstruction problem is in general NP-hard if the number of projections is more than two [5]. Efficient algorithms only exist for some special classes of images (see, e.g., [6, 7]). This means that we usually cannot hope to gain perfect reconstructions in reasonable time, and most reconstruction algorithms are only well-constructed heuristics, which approximate the solution.

Despite the problems mentioned above, our aim is to get reconstructions as accurate as possible, and develop robust algorithms which can handle computational problems, and the possible defects of the measured data sets.

In this paper, we give an approach for describing the uncertainty of the reconstructions in discrete tomography, and provide a method that can measure the information content of the projections in the binary case. Our approach is capable of approximating (on a grid based representation) the uncertainty of each pixel of the reconstructed image separately. It measures how each part of the reconstruction of the object is determined by the given projection data, and provides a local reliability measure for the parts of the reconstruction.

This measurement is unique in the literature, as to the best of our knowledge related contributions only exist for measuring the overall reliability of reconstructions. For example, in [8] the authors gave an upper bound on the difference between the possible binary reconstructions of an object, which provided a measure of the variability of reconstructions from a given projection set. Our approach takes one step further by approximating the reliability of each part of the reconstruction, separately.

Such methods can be useful, e.g., in the nondestructive testing of objects to sort out false results. For example, if one uses discrete tomography for detecting small fractures in industrial parts, small errors in the reconstruction can lead to false conclusions. In this case our algorithm can be used together with the reconstructed image, to check the reliability at specific parts of the recon- 
struction.

The structure of the paper is the following. In Section 2 we give a brief explanation of the reconstruction problem, and its algebraic based formulation. In Section 3, we explain in more detail the uncertainty problem arising in the field of discrete tomography. In Section 4 we provide an algorithm, that - with the right parameter setting is capable of measuring uncertainty of the projection data. In Section 5 we outline a test frame set that was used for validating our method, and provide some results. Finally, Section 6 is for the conclusion.

\section{Algebraic Formulation of Discrete Tomog- raphy}

We present our results for the two-dimensional case of discrete tomography, but the described methods can be extended to higher dimensions in a straightforward way.

In this paper, we will use the algebraic formulation of discrete tomography, and assume that the object to be reconstructed is represented on a two dimensional image of size $n$ by $n$. Also, we will assume a parallel beam projection geometry with each projection value given by the integral of the image on a straight line.

With these assumptions the discrete reconstruction problem can be written in a form of a linear equation

$$
\mathbf{A} \mathbf{x}=\mathbf{b}, \quad \mathbf{x} \in L^{n^{2}},
$$

where

- $\mathbf{x}$ is the vector of all $n^{2}$ unknown image pixels,

- $m$ is the total number of projection lines used,

- $\mathbf{b}$ is the vector of all $m$ measured projection values,

- A is a projection coefficient matrix, that describes the projection geometry by all $a_{i j}$ elements giving the length of the line segment of the $i$-th projection line through the $j$-th pixel,

- and $L=\left\{l_{0}, l_{1}, \ldots, l_{c}\right\}$ is the set of the possible intensities (assuming that $l_{0}<l_{1}<\ldots<l_{c}$ ). In the binary case $L=\{0,1\}$.

An illustration of the parallel beam geometry is given in Figure 1.

With the above formulation, one can acquire a solution of the reconstruction problem by solving (1).

\section{The Uncertainty Problem}

In a mathematical sense, the algebraic formulation of discrete tomography means that we have a search space with $n^{2}$ dimensions (as many dimensions as the number of pixels on the image to be reconstructed). In this search space the equation system $\mathbf{A x}=\mathbf{b}$ determines an $\mathcal{H}$ hyperplane,
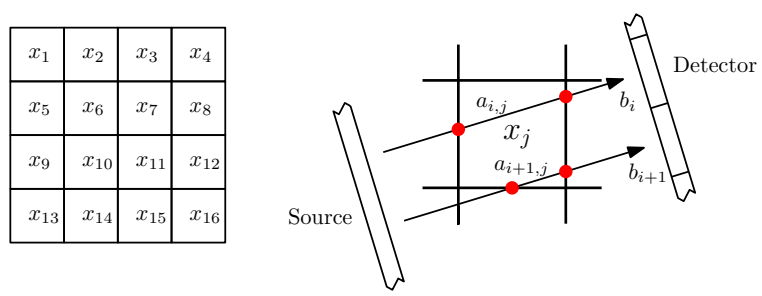

Figure 1. Representation of the parallel beam geometry on a discrete image.

which has $\left(n^{2}-\operatorname{Rank}(\mathbf{A})\right)$ dimensions. Also, the knowledge, that we are looking for discrete solutions gives us a finite set of possible discrete points of $\mathcal{D}=L^{n^{2}}$.

In the ideal case, the feasible reconstructions belong to the $\mathcal{H} \cap \mathcal{D}$ intersection. These are the images that satisfy the projections, and the discreteness criteria.

In many practical applications the situation is more complicated. First of all, the models used for describing the reconstruction problem and the projection acquisition methods are not perfect yielding changes of the position of the $\mathcal{H}$ hyperplane. In this case the $\mathcal{H}$ hyperplane of solution shift from its ideal position, and we can only get an approximation of the ideal plane of real solutions. In extreme cases, we can get an inconsistent equation system, that has no solutions at all.

Taking many projections, on the other hand, as described above, might also conflict with limitations, since the projection acquisition can have a high cost, or unwanted effects on the object of study. This will yield a really extensive $\mathcal{H}$ hyperplane of solutions.

Nevertheless, in practical applications, there is an actual object of study we want to reconstruct, and all other results would be considered incorrect. This can leave us with a problem, where we want to find a reconstruction from an incomplete, and incorrect data set.

In this case it would be more fortunate to view the reconstruction problem in a probabilistic context, where each discrete valued solutions $\mathbf{x} \in L^{n^{2}}$ has a

$$
P(\mathbf{x} \mid \mathbf{A}, \mathbf{b})
$$

probability of being the correct one. Naturally the closer an $\mathbf{x}$ solution is to the hyperplane determined by $\mathbf{A x}=\mathbf{b}$ (or if the system of equations is inconsistent, then the more the solution satisfy the projections), the higher its probability should be.

With this, we can calculate for each $i$-th pixel, the probability of that pixel taking a specific $v$ value in the correct solution. This is given by

$$
P\left(x_{i}=v \mid \mathbf{A}, \mathbf{b}\right)=\sum_{\substack{\mathbf{y} \in L^{n^{2}} \\ y_{i}=v}} P(\mathbf{y} \mid \mathbf{A}, \mathbf{b}) \quad v \in L .
$$


Furthermore, we can compute the entropy of each pixel as

$$
\begin{aligned}
& H_{i}\left(x_{i}\right)= \\
& \sum_{k=0}^{c}\left(-P\left(x_{i}=l_{k} \mid \mathbf{A}, \mathbf{b}\right) \cdot \log _{2}\left(P\left(x_{i}=l_{k} \mid \mathbf{A}, \mathbf{b}\right)\right)\right) .
\end{aligned}
$$

This way we can measure the uncertainty of each pixel. We should note that this value is based only on the parameters of the projections, and the information content of the measured projection data. Pixels with high entropy values are ambiguous, and one cannot hope to get a reliable reconstruction of them without additional information. The projections simply do not hold enough data, to determine these pixels.

For all the pixels, the values of (3) can be arranged into coefficient probability maps that show the likelihood of each pixel to belong to a specific intensity. Also, the values of (4) together give an uncertainty map, that describes the uncertainty of the areas of the reconstruction based on the projection data. Thus we can get a picture of the information reliability of the projection set and the reconstructions.

Unfortunately, even if we can describe the probabilities belonging to the projection data, the exponential number of possible discrete solutions makes it hard to compute this type of uncertainty measurement. In the next section we describe a method for approximating this value in the binary case, and with the aid of that to measure the information content of the projection data.

\section{Approximating Local Uncertainty in Bi- nary Reconstructions}

In [9], the authors proposed an algorithm for discrete tomography, that is capable of reconstructing images by minimizing an energy function. Here, we give a modified version of that algorithm, that can approximate the pixel uncertainties of binary reconstructions, by producing a "least binary" result.

\subsection{Algorithm for Approximating Pixel Uncertainty}

The algorithm is based on minimizing an energy function of the form

$$
\mathcal{E}(\mathbf{x})=\frac{1}{2}\|\mathbf{A} \mathbf{x}-\mathbf{b}\|_{2}^{2}+\mu \cdot g(\mathbf{x}),
$$

where $\mathbf{A}, \mathbf{b}$ and $\mathbf{x}$ are as defined in Section $2, g(\mathbf{x})$ is a function holding information of the discreteness of the reconstruction, and $\mu$ is the weight of the discreteness prior.

The first $\|\mathbf{A x}-\mathbf{b}\|_{2}^{2}$ term is responsible for the projection correctness. It takes its minimal values where the solution best satisfies the projections. In the ideal case, these solutions would be the points of $\mathcal{H}$ defined in Section 3, but in case of an inconsistent equation system, reconstructions best fitting to the projections will give minimal $\|\mathbf{A x}-\mathbf{b}\|_{2}^{2}$ values.
In some similar energy minimization based reconstruction methods $[9,10]$, the $g(\mathbf{x})$ is a discretizing term taking its minimal values in discrete points, thus propagates discrete solutions. Here, we would rather call this term a discreteness prior and emphasize, that it is not necessary to propagate discrete solutions with it. In fact, with the different choice of $g(\mathbf{x})$ one can reach different effects on the result, and gain different kinds of information on the reconstructions.

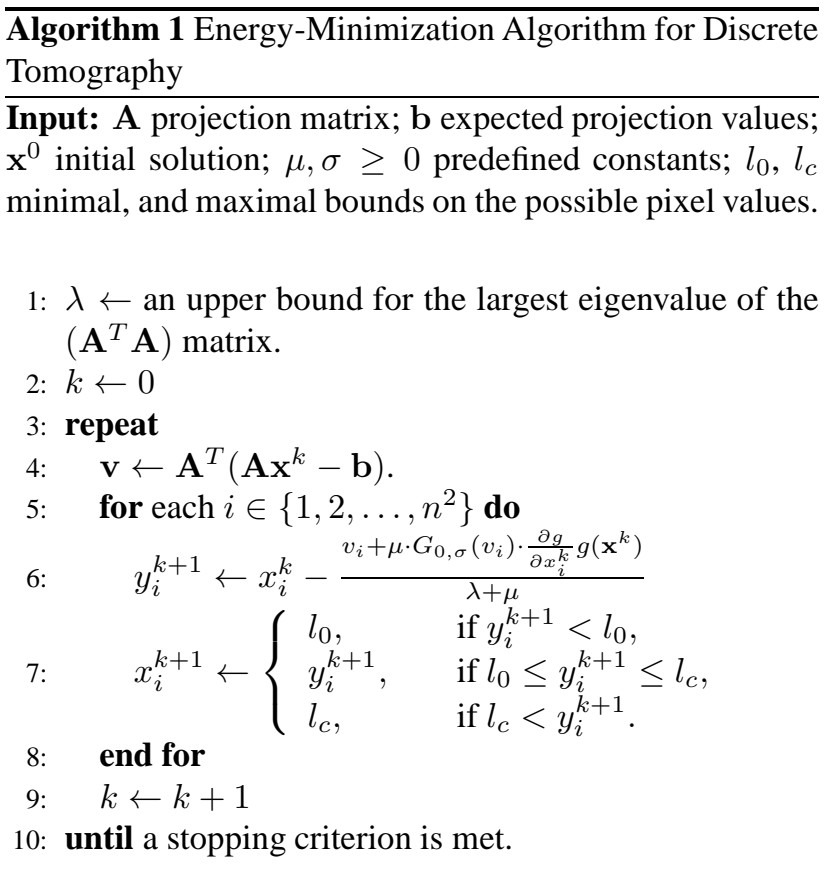

The formal description of the optimization process is given in Algorithm 1. The basic idea of the optimization process is to distinguish between the priorities in the energy function. The core of the whole optimization process is a simple gradient method with an automatic weighting between the two terms. The algorithm starts from an arbitrarily defined starting point. Then, in each iteration step, we set the weight of the discreteness term according to the projection correctness.

Note, that in each iteration step the gradient of the $\frac{1}{2}\|\mathbf{A x}-\mathbf{b}\|_{2}^{2}$ projection correctness term gives for each pixel a measurement of how much that specific pixel should be modified to get a correct reconstruction. These values can also be understood as the backprojected error of the projections of the current solution. If, for a pixel, this value is close to 0 , then that specific pixel does not really take a part in distorting the projections. On the other hand, if the gradient on a pixel is big in absolute value, then the pixel probably needs further adjustment to reach an acceptable solution.

In Algorithm 1 this is used to steer the optimization process. We apply a $G_{0, \theta}(z)=\exp \left(\frac{z^{2}}{\theta^{2}}\right)$ unnormalized Gaussian function to the per-pixels backprojected errors, and use the resulting values to weight the discretizing term on each pixel. With this, if the a pixel value is considered to 
be settled, then the discreteness prior gets a higher weight, and thus it starts to steer the result to a desired point.

We should also note that the original reconstruction algorithm described in [9] contained an additional smoothness prior, that can be useful in the reconstruction of objects. In this case, we do not need the smoothness prior, since we are trying to measure the information content of the projections themselves. Measuring the combined information of the projection data, and some extra prior knowledge can be subject of further studies.

\subsection{Approximating Pixel Uncertainty in Binary To- mography}

Based on the argument of Section 3 and the algorithm given above, we defined a way for the simple approximation of the pixel uncertainty in case of binary tomographic reconstructions.

The basic idea of our concept is to find the reconstruction that satisfies the acquired projection data, but contains the least discrete pixel values, i.e., in which the pixel intensities are the farthest away from the the binary values. In that way we can measure the relation between the projection data, and the fact that we are looking for binary solutions, and approximate how easy it is to move each pixel value away from the binary domain. This can give information about the uncertainty of a projection set itself, even if the original image and the entire search space are unknown.

With Algorithm 1 this can be done by setting a discreteness prior that discourages binary, or close to binary pixel values. One such prior can be given by

$$
g(\mathbf{x})=\frac{1}{2} \cdot\left\|\mathbf{x}-\frac{1}{2} \cdot \mathbf{e}\right\|_{2}^{2},
$$

where e stands for a vector with all $n^{2}$ positions having a value of 1 . Note, the "upside-down" version of this discreteness prior have been used in previous works for finding binary solutions of the reconstruction problem.

Also note, that - although the projection correctness term has a higher priority in the algorithm than the discreteness prior - the algorithm uses a weighting between the two terms of the energy function, and in the end the acquired solution is not guaranteed to strictly satisfy the projections, only approximates them.

When the result is computed, taking the entropy

$$
H_{i}\left(x_{i}\right)=-\left(x_{i} \cdot \log _{2}\left(x_{i}\right)+\left(1-x_{i}\right) \cdot \log _{2}\left(1-x_{i}\right)\right),
$$

for each pixel value, should give an approximation of the pixel uncertainty given in (4).

Finally, it would be possible to use different types of algorithms for approximating the coefficient probability, and uncertainty maps. Such algorithms should be aiming to find solutions, which satisfy the $\mathbf{A x}=b$ equation system, and are as close to the $\left(\frac{1}{2}, \frac{1}{2}, \ldots, \frac{1}{2}\right)^{T}$ vector as possible (i.e., solutions being closest to the least binary image possible). Investigating such algorithms is a subject to our further studies.

\section{Validation and Results}

For the validation of the measurement we performed software tests on some phantom images. We took a set of binary images, produced their projections and tried to measure the uncertainty of the pixels with our approach.

We also needed another method, that can produce the local uncertainties of the reconstructions, to compare our proposed algorithm with. Unfortunately, to the best of our knowledge, the literature does not contain such algorithms. Therefore, we have decided to sample the space of reconstructions in order to approximate the probabilities of (2).

\subsection{A Stochastic Approximation of Pixel Uncertainties}

For the random sampling of the search space we performed several reconstructions from the same projection data with a randomized reconstruction algorithm. With this, we could get random elements of the space of feasible reconstructions and gain statistics on the pixel intensities. We have chosen to use the Simulated Annealing based reconstruction method described in [11]. The slightly modified code of this algorithm is given in Algorithm 2.

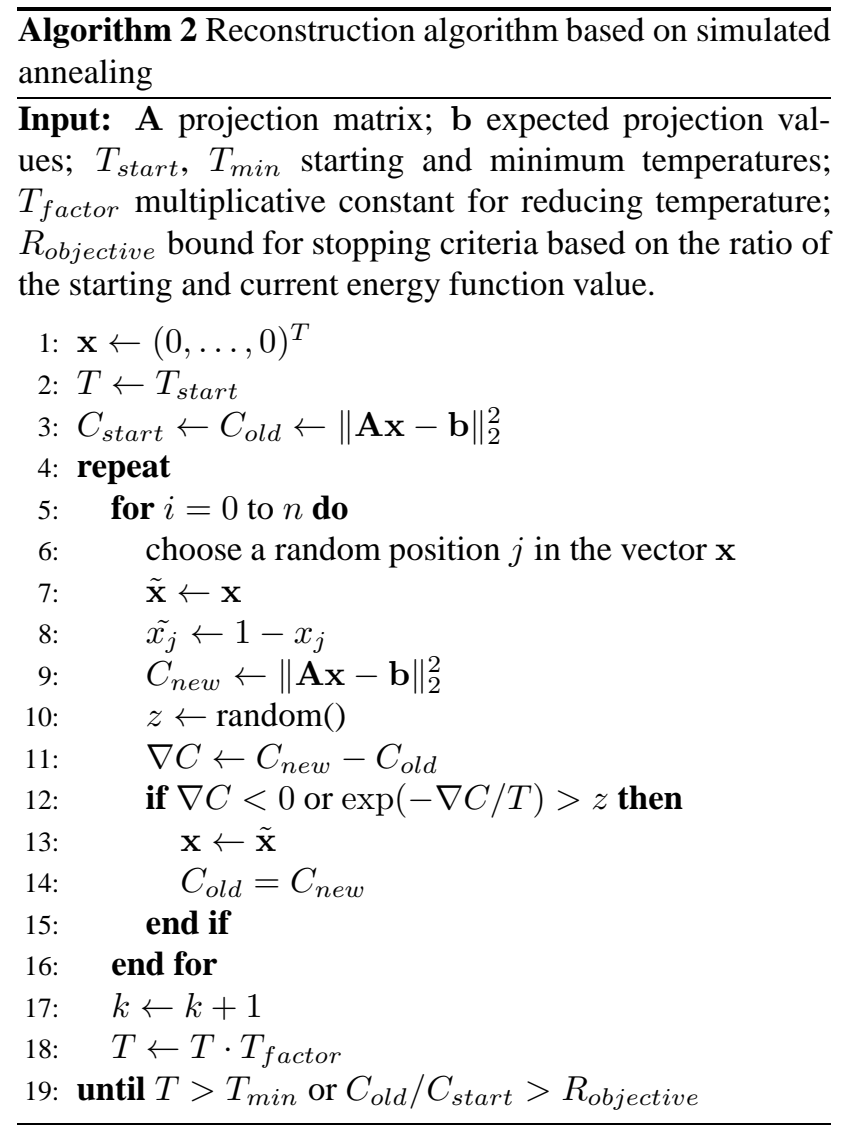

With the proper parameter settings, and an unlimited iteration count, Simulated Annealing based methods should converge to an optimal binary solution. Practically, such a process would be impossible to carry out, and Algorithm 2 is a heuristic method for approximating a correct 


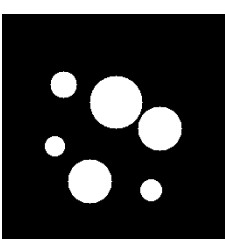

a)

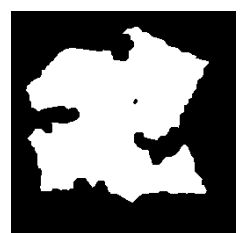

b)

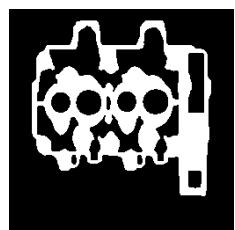

c)
Figure 2. Some of the software phantoms used for testing.

solution.

Because of the stochastic nature of this process, with each run of Algorithm 2 we get a random element of the search space. Reconstructions better satisfying the projections will have a higher probability to be found, and these probabilities should correspond to the probabilities given in Section 3. Therefore, by running this algorithm several times we can get a faithful sampling of the search space, and averaging the pixel values would provide the required probabilities.

Note, that the original energy function of this algorithm also contained a smoothness regularization, but in this case we omitted this term to get reconstructions which only rely on the projections themselves.

\subsection{Test Frame Set}

In the evaluation of the method we took a set of phantom images, produced their projection sets with different numbers of projections, and computed the pixel uncertainties from the given data with the two methods described above. Unfortunately, the validation method given in Section 5 had an enormous time requirement, and we could only test our methods for 3 images, which were chosen carefully based on our previous experiences. These phantoms can be seen in Figure 2. Further validation is among our future plans.

For performing the computation, the parameters of Algorithm 1 were set empirically. We used the initial $\mathbf{x}^{0}=$ $(0.5, \ldots, 0.5)^{T}$ vector in the beginning of the optimization process, and chosen the values $\mu=1$ and $\sigma=0.25$. The iteration was stopped when the difference between the solutions of the $k$-th and $(k+1)$-th iteration steps computed as $\left\|\mathbf{x}^{k+1}-\mathbf{x}^{k}\right\|_{2}$ became less then 0.001 or the number of iterations reached a limit of 5000 .

As for the parameters of the simulated annealing based method, we used the parameter settings as described in [11], except, that we did not apply a smoothness regularization term in the process. More exactly, the parameter values were $T_{\text {start }}=4.0, T_{\text {min }}=10^{-14}, T_{\text {factor }}=0.97$, $R_{\text {objective }}=10^{-5}$. Moreover, for each given projection set we averaged 100 runs of the optimization process to approximate the probability maps given in Section 3 .

The implementation of Algorithm 1 was coded in C++ with GPU acceleration with the Nvidia CUDA sdk. Algorithm 2 on the other hand was not suited for parallel implementation and GPU acceleration, and it was coded in
MATLAB.

After producing the uncertainty maps from the results of Algorithm 1 and Algorithm 2 with the projection sets, we compared the results given by the two methods visually, and calculating the average pixel difference

$$
R(\mathbf{x}, \mathbf{y})=\frac{1}{n^{2}} \sum_{i=1}^{n^{2}}\left|x_{i}-y_{i}\right|
$$

This measure takes values between 0 and 1 . For any pair of $\mathbf{x}$ and $\mathbf{y}$ images, if there is a correspondence between the pixel pairs of $\mathbf{x}$ and $\mathbf{y}$, then the difference between the pixels at the same positions should be small and the $R(\mathbf{x}, \mathbf{y})$ will take a value close to 0 . If the correspondence is weaker, then this average difference will lean towards 1.

\subsection{Results}

At the different steps of the algorithms, we got different types of results. First, at the end of the optimization process of Algorithm 1 - and after averaging 100 results of Algorithm 2 - we got continuous reconstructions approximating probability maps of Section 3. Second, when applying (7) to the pixels of the approximate probability maps, we got uncertainty maps of the reconstructions, showing for each pixel its vagueness with the given projection data. Some of the results can be seen in Figure 3.

We also compared the data contained by the two types of images by the calculating the average pixel difference given in (8). Some of the resulted data can be seen in Table 1.

In the images of Figure 3 we can see that there is no significant difference between the coefficient probabilityand uncertainty- maps computed by the two compared methods. This is in accordance with the calculated average differences in Table 1, where the values close to 0 indicate a strict correspondence between the two types of uncertainty measures.

The time requirement of our proposed algorithm was about 10-20 seconds for measuring each uncertainty maps on a PC with an Intel Q9500 CPU and an Nvidia GTX250 GPU. On the other hand, running the simulated annealing based algorithm 100 times (with the same CPU, but without GPU acceleration) for measuring probabilities took about 2 days for each image and projection number.

As a conclusion we can say that the two approaches for measuring the local uncertainties provide the same information, and are capable of approximating the local reliabilities of the reconstruction. However, the high time requirement of the Simulated Annealing based approach makes it impractical for applications. Fortunately, the Simulated Annealing-based method was only used for validation purposes, and our energy minimization based proposed algorithm can give results in reasonable time. 
Original image \# projs. frobability map

from Alg. 1 from Alg. 2

Uncertainty map

from Alg. 1

from Alg. 2
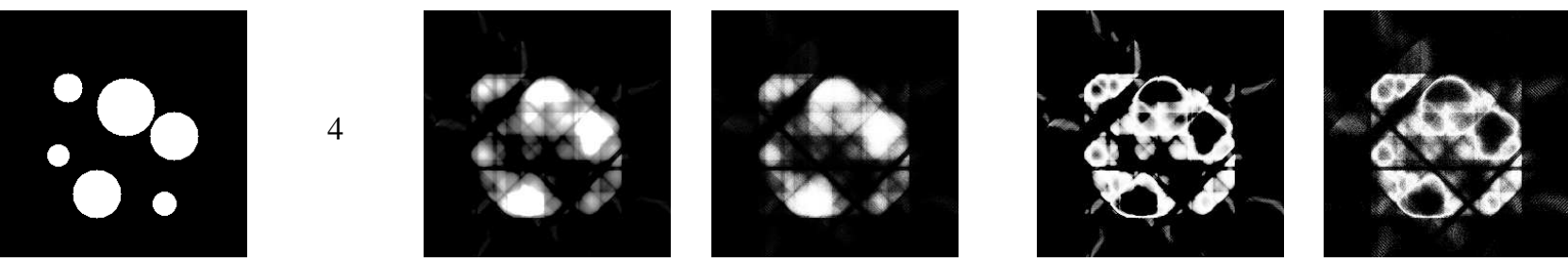

5
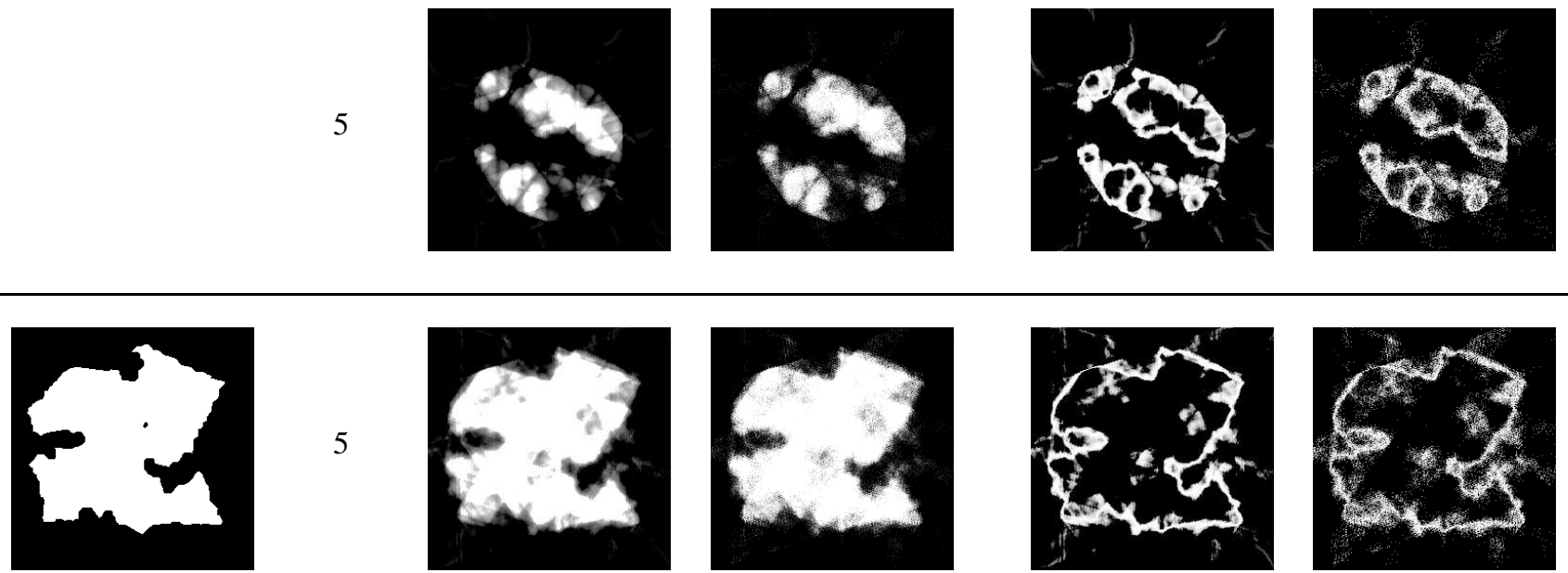

6
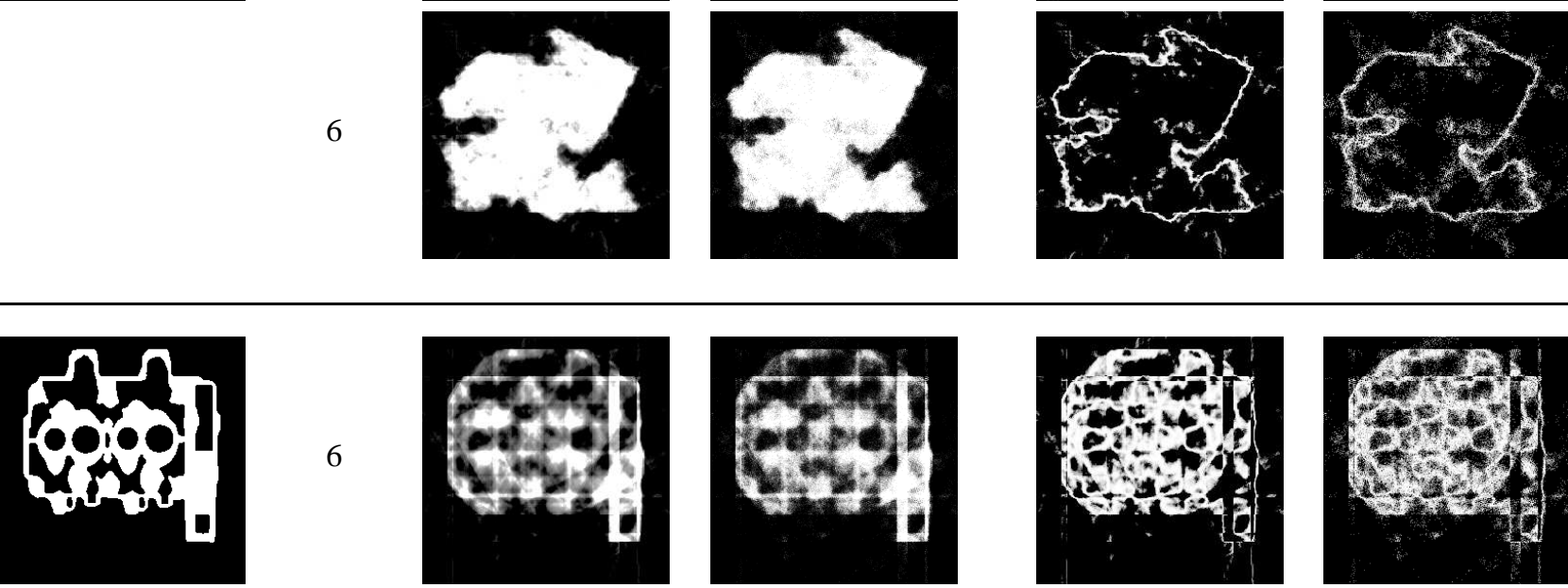

9
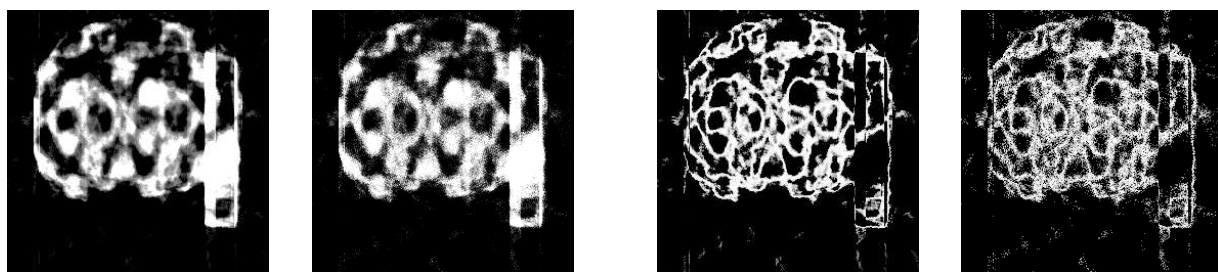

Figure 3. Coefficient probability-, and uncertainty-maps produced by Algorithm 1 and Algorithm 2. On the coefficient probability maps: white areas should with high probability take the intensity value 1, and black areas are with high probability 0 . Intensity values belonging to gray areas are not determined by the projections. On the uncertainty maps: Dark areas are determined by the projections, while white areas are not, and hold uncertainty. 
Table 1. Average pixel differences between the probability and uncertainty maps given by the two uncertainty measurement methods, according to test images and projection numbers.

\begin{tabular}{|c||c|c|c|}
\hline \multicolumn{4}{|c|}{ Difference between the probability maps } \\
\hline \# projs. & Figure 2a & Figure 2b & Figure 2c \\
\hline \hline 2 & 0.021 & 0.033 & 0.034 \\
\hline 3 & 0.020 & 0.038 & 0.040 \\
\hline 4 & 0.016 & 0.022 & 0.032 \\
\hline 5 & 0.018 & 0.026 & 0.035 \\
\hline 6 & 0.014 & 0.019 & 0.033 \\
\hline 9 & 0.007 & 0.013 & 0.033 \\
\hline 12 & 0.005 & 0.008 & 0.025 \\
\hline 15 & 0.004 & 0.006 & 0.027 \\
\hline 18 & 0.003 & 0.005 & 0.021 \\
\hline
\end{tabular}

\begin{tabular}{|c||c|c|c|}
\hline \multicolumn{4}{|c|}{ Difference between the uncertainty maps } \\
\hline \# projs. & Figure 2a & Figure 2b & Figure 2c \\
\hline \hline 2 & 0.038 & 0.088 & 0.069 \\
\hline 3 & 0.053 & 0.095 & 0.084 \\
\hline 4 & 0.046 & 0.065 & 0.073 \\
\hline 5 & 0.049 & 0.078 & 0.076 \\
\hline 6 & 0.046 & 0.066 & 0.082 \\
\hline 9 & 0.030 & 0.051 & 0.088 \\
\hline 12 & 0.022 & 0.040 & 0.080 \\
\hline 15 & 0.016 & 0.030 & 0.088 \\
\hline 18 & 0.013 & 0.021 & 0.076 \\
\hline
\end{tabular}

\section{Conclusion}

We gave a practical description of the data uncertainty problem arising from the field of discrete tomography, providing a measure for the binary case, that can approximate the local uncertainties of the reconstructed image.

Given the projections of a homogeneous object, we provided a way to approximate how likely will each pixel of the reconstructed image take a 0 or 1 value on a correct reconstruction. With this, one can approximate the uncertainty of each pixel, get a picture of the information content of a projection set provided for a reconstruction, and measure how each part of the reconstructed image is determined by the given projections. This information can be useful in practical applications to measure the accuracy and reliability of the reconstructed results.

In our future work we plan to perform further validation of our local uncertainty measure, to extend the results to the non-binary case of discrete tomography (i.e., when there are more than two possible intensities in the reconstructed images), and to try our reconstruction in practical applications. Also, we are making efforts to summarize the local uncertainties to one global measurement, that can describe the overall information content of a projection set, and reveal connections to the method described in [8].

\section{Acknowledgements}

The work of László G. Nyúl was supported by the János Bolyai Research Scholarship of the Hungarian Academy of Sciences. The work of Péter Balázs was supported by the OTKA PD100950 grant of the National Scientific Research Fund.

\section{References}

[1] A.C. Kak, M. Slaney, Principles of computerized tomographic imaging, IEEE Press, New York, 1999.

[2] G.T. Herman, Fundamentals of Computerized Tomography, Image Reconstruction from Projections, 2nd edition, Springer-Verlag, London, 2009.

[3] G.T. Herman, A. Kuba (Eds.), Discrete Tomography: Foundations, Algorithms and Applications, Birkhäuser, Boston, 1999.

[4] G.T. Herman, A. Kuba (Eds.), Advances in Discrete Tomography and Its Applications, Birkhäuser, Boston, 2007.

[5] R.J. Gardner, P. Gritzmann, Discrete tomography: Determination of finite sets by X-rays, Trans. Amer. Math. Soc. 349(6), pp. 2271-2295 (1997).

[6] S. Brunetti, A. Del Lungo, F. Del Ristoro, A. Kuba, M. Nivat, Reconstruction of 4- and 8-connected convex discrete sets from row and column projections, Lin. Alg. Appl. 339, pp. 37-57 (2001).

[7] M. Chrobak, C. Dürr, Reconstructing $h v$-convex polyominoes from orthogonal projections. Information Processing Letters 69(6), pp. 283-289 (1999).

[8] K.J. Batenburg, W. Fortes, L. Hajdu, R. Tijdeman, Bounds on the difference between reconstructions in binary tomography, Discrete Geometry and Computer Imaginary, LNCS 6607, 369-380 (2011).

[9] L. Varga, P. Balázs, A. Nagy, An energy minimization reconstruction algorithm for multivalued discrete tomography, 3rd International Symposium on Computational Modeling of Objects Represented in Images, Rome, Italy, Proceedings (Taylor \& Francis), 179185 (2012).

[10] T. Schüle, C. Schnörr, S. Weber, J. Hornegger, Discrete tomography by convex-concave regularization and D.C. programming, Discrete Applied Mathematics 151, 229-243 (2005).

[11] S. Weber, A. Nagy, T. Schüle, C. Schnörr, A. Kuba, A benchmark evaluation of large-scale optimization approaches to binary tomography, Lecture Notes in Computer Science, vol. 4245, 146-156 (2006). 